\title{
Planificación urbana y espirovías en la "Perla del Pacífico": algunas notas sobre Valparaíso en el decenio 1930.
}

City Planning and "espirovías" in the "Pearl of the Pacific" (Valparaiso): several notes on Valparaiso from the 1930's.

María Isabel Pavez R.

\section{Filiación}

Académica de la Facultad de Arquitectura y Urbanismo de la Universidad de Chile mpavez@uchilefau.cl

\section{Resumen}

Se aporta algunas notas para la historiografía en Urbanismo, Planificación y Diseño Urbano en Chile y en Valparaíso en los años 1930, dando cuenta de la capacidad reflexiva y creativa de los profesionales precursores.

\section{Palabras Clave}

Valparaíso 1930, Karl Brunner, Jacques Lambert, Luis Muñoz Maluska, Agostino Bastiancig, planificación urbana, diseño urbano, espirovías.

\section{Abstract}

The article features several notes on the history of City Planning and Urban Design in Chile and Valparaiso in the 1930's thus giving insights about the creative and reflexive qualities of the forefathers of Urban professionals.

\section{Key words}

Valparaíso 1930, Karl Brunner, Jacques Lambert, Luis Muñoz Maluska, Agostino Bastiancig, urban planning, urban design, espirovías.

\section{Sumario}

1.- Karl Brunner, Valparaíso, y la Sección de Urbanismo en 1929

2.- "Anteproyecto Plano Oficial de Urbanización" de Valparaíso desde la Sección de Urbanismo en 1931

3.- Jacques Lambert en Chile, en 1929

4.- El concurso del Alcalde Rosas para la transformación de Valparaíso

5.- Labor del Instituto de Urbanismo de Valparaíso

6.- Valparaíso se renueva o muere: proposición de "espirovías" en la Perla del Pacífico Palabras finales

\section{1.- Karl Brunner, Valparaíso, y la Sección de Urbanismo en 1929}

Luego que el 27 de noviembre de 1929, el arquitecto y urbanista austriaco Dr. Karl Brunner primer Asesor de la Sección de Urbanismo del Departamento de Arquitectura de la D.G.O.P. del Ministerio de Fomento de Chile [MOP] recién creada visitó la ciudad de Valparaíso acompañado de las autoridades locales, expresó que el modo de pensar moderno sobre el Urbanismo comprendía no sólo el estudio de los aspectos artísticos y estéticos, sino también de los económicos y sociales de las obras. 
Comentó Brunner sobre la vialidad de Valparaíso:

El problema de urbanización de Valparaíso es "sui generis", por cuanto el puerto presenta una escasa superficie plana y la ladera de los cerros no es continua, sino que interrumpida por numerosas quebradas que los aíslan unos de otros. En el sentido lógico de la urbanización, cada cerro debe constituir un centro. Pero esta forma de urbanizar sería costosa, de manera que la forma más práctica es la de buscar un buen medio de comunicación. En Valparaíso se ha encontrado en el Camino de Cintura. Las ciudades, para desarrollarse, necesitan del comercio que siempre busca las calles largas y anchas. Es preciso entonces dotar a los cerros de centros comerciales de aprovisionamiento para impulsar su desarrollo. En este sentido, el Camino de Cintura será una gran arteria, y contribuirá de un modo eficiente a la urbanización de los cerros.

Estimó Brunner que en Chile existía un alto grado de cultura urbana. Los estudios técnicos se hallaban muy bien dirigidos en su opinión, y la Universidad formaba profesionales de gran preparación y competencia. Como complemento de esa obra educativa, Brunner dictaría un postítulo en Urbanismo, donde todos los participantes tendrían que integrar un taller de proyectos.

Contándose con Brunner, y siendo el arquitecto Luis Muñoz Maluschka el principal interlocutor de la contraparte, se revisó la Ordenanza de 1930, la que contenía disposiciones sobre materias no incluidas en la Ley 4.563. El 20 de mayo de 1931 se aprobó el D.F.L. N0345 con la nueva Ley General sobre Construcciones y Urbanización, y su Ordenanza ajustada ${ }^{2}$. Esta ley creó las Direcciones de Obras en todos los Municipios de la República, la obligatoriedad de tener "Planos Oficiales de Urbanización" en todas las comunas de más de 8.000 habitantes, y "zonas industriales" en todas las ciudades de más de 20.000 habitantes. En adelante, las Municipalidades deberían procurar que los barrios obreros contaran con los establecimientos de educación y asistencia social. Calles, plazas y plazoletas podrían ser exigidas, representando hasta un $30 \%$ de la superficie a urbanizar. De esa superficie, hasta un $7 \%$ podría ser requerido para parques, jardines y otros espacios públicos ${ }^{3}$.

La Sección de Urbanismo inició proyectos de Planos Reguladores y urbanizaciones diversas en Arica, Iquique, Antofagasta, Tocopilla, la Serena, Coquimbo, Valparaíso, Viña del Mar, San Antonio, Talca, Puerto Varas, Población Río Ibáñez, Población Navarino, y algunos pueblos menores ${ }^{4}$.

\footnotetext{
1 EL MERCURIO, Valparaíso, 27 nov. 1929. "El problema de urbanizar a Valparaíso tiene características propias que impiden seguir los métodos de otras ciudades".

INSTITUTO NACIONAL DE INVESTIGACIONES TECNOLOGICAS Y NORMALIZACIÓN, C.D.U. $69.05+711$, 10 de nov. de 1949, Ley y Ordenanza General sobre Construcciones y Urbanización. [Contiene compilación histórica de legislación precedente].

3 Ibidem.

4 CHILE - DIRECCION GENERAL DE OBRAS PÚBLICAS. 1930. Memoria anual correspondiente al año 1929, República de Chile, Talleres de la Editorial Nascimiento, 1930, 176 págs.
} 


\section{2.- "Anteproyecto Plano Oficial de Urbanización" de Valparaíso desde la Sección de Urbanismo en 1931}

La buena impresión que había tenido Brunner en sus dos estadas en Chile entre 1929 y 34, fue confirmada por el norteamericano Francis Violich en la segunda mitad del decenio de 1930, y registrada en su libro Cities of Latin America. Housing and planning to the south, publicado en Nueva York en $1944^{5}$. Luego de recorrer los países latinoamericanos en busca de los hombres "punta de lanza" de la planificación urbana, refirió en su libro que en Chile había encontrado un grupo de técnicos con avanzados conocimientos en la especialidad. En primer lugar, el Arqto. Luis Muñoz Maluschka, cuya formación y práctica básica había sido obtenida en Alemania; su concepción de la planificación se basaba en "una comprensión muy realista de los factores económicos tanto como de los físicos"6.

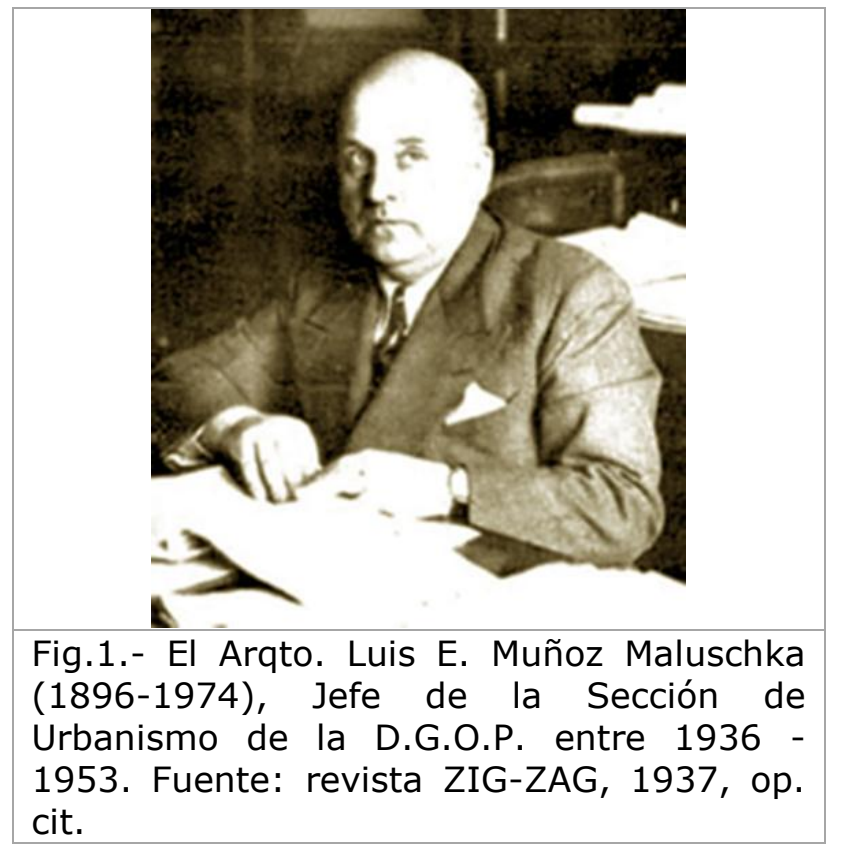

En la Sección de Urbanismo de la D.G.O.P., Violich pudo apreciar la labor de planificación de Valparaíso en el plano "VALPARAISO - D.G.O.P. - Sección de Urbanismo - Anteproyecto Plano Oficial de Urbanización - Plano de Zonificación " (Esc. 1:5.000). Este anteproyecto, firmado por Muñoz Maluschka, Jefe de la Sección, comenzó a prepararse desde el momento mismo de la creación de esta última en 1929, y operó oficialmente recién luego de la publicación en el Diario Oficial, en 1936, del D.F.L. 345 aprobado en 1931, siendo publicado en blanco y negro en el libro de Violich citado.

\footnotetext{
5 VIOLICH, Francis. Cities of Latin America. Housing and planning to the south. Nueva York: Reinhold Publishing Corporation, 1944. 241 p.

6 Otras personalidades destacadas nombradas fueron los Arqtos. Rodulfo Oyarzún, Ricardo González, Federico Oehrens, Roberto Humeres, y Héctor Vigil. Este último profesional cumplió su misión desde el Instituto de Urbanismo de Valparaíso fundado en 1933.
} 


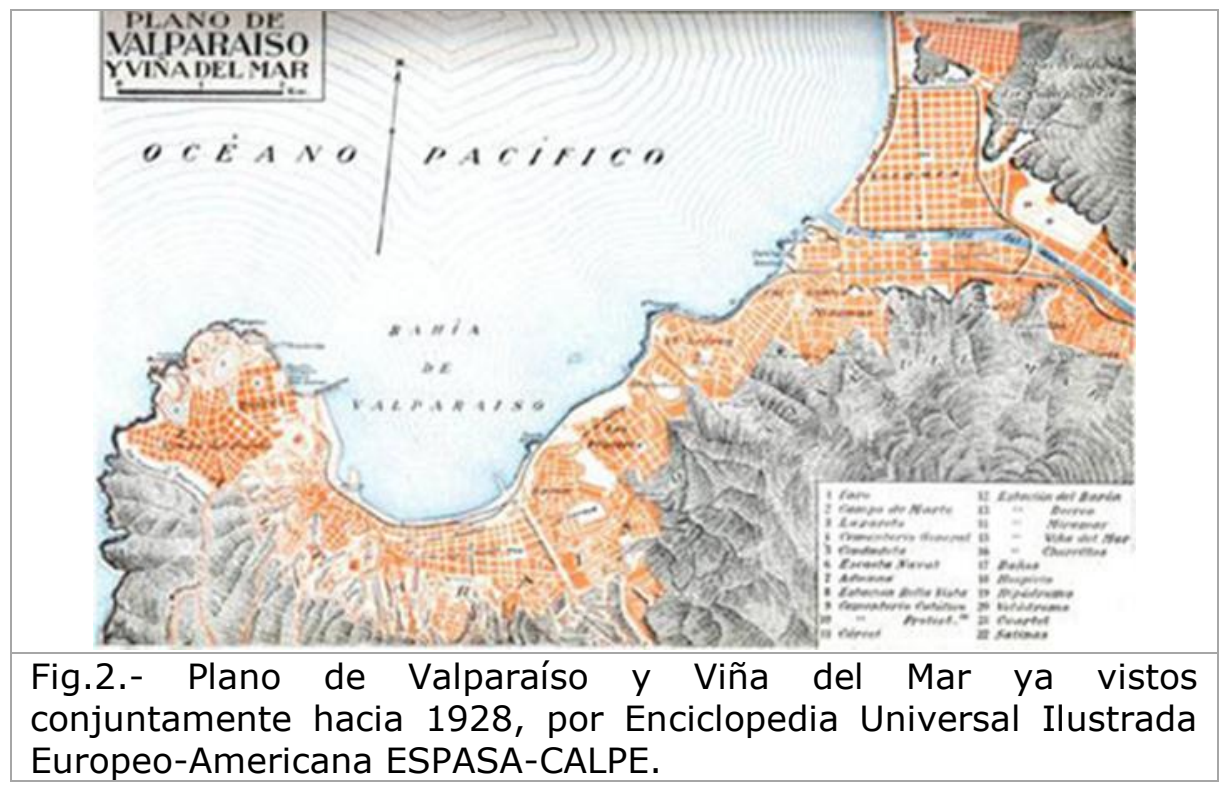

Cabe destacar que, ya en la época, la Muñoz Maluschka tenía claro el problema intercomunal de Valparaíso-Viña del Mar, y también su participación en una macroregión central. Observamos, en primer lugar, que en lo referido al concepto del "Plan Territorial" difundido por este urbanista mediante el cual se relacionaba los diversos núcleos poblados y la economía del territorio este instrumento debía comprender un "Programa de Acción", y las directrices para el establecimiento de los "Planes Regionales". En el caso del territorio chileno, Muñoz Maluschka planteó la necesidad de considerar máximo 8 regiones, las que debían organizar sus distintas comunas y núcleos poblados con la finalidad de elaborar en común sus planos reguladores ${ }^{7}$.

La ciudad de Valparaíso quedaba integrada, en esta ponencia, a una "3a Región" comprendiendo: "Santiago y sus satélites, Valparaíso y sus satélites, y San Antonio y Rancagua".

En la voluntad de promover en Chile una cultura de asociatividad -la que a fines del siglo XX reapareció como novedad reciente-, reiteró Luis Muñoz en 1936 sus ponencias de $1934^{8}$, conforme a los conceptos transferidos por él mismo desde Alemania:

Mientras los diversos municipios no comprendan y dominen los problemas económicos intercomunales que los ligan y que, dentro de la incomprensión existente sigan combatiendo y anulándose recíprocamente, no habrá progreso integral y sólo prosperará la región o ciudad favorecida temporalmente por alguna influencia política pasajera.

\footnotetext{
7 MUÑOZ MALUSCHKA, Luis. 1936. "Planos Reguladores y zonificación territorial". En: revista ARQUITECTURA, Santiago de Chile, Editorial Antares, enero de 1936, №4.

8 Ibidem.
} 
No obstante carecerse aun legalmente del instrumento específico de planificación de nivel intercomunal, se pudo contar desde $1936^{\circ}$ con proyectos indicativos en este escalón territorial. Estas proposiciones indicativas pueden considerarse oficiales, en virtud del Art. 4 de la Ley de Municipalidades chilena, vigente desde 1936, señalando la posibilidad de resolver en común con otros municipios, los problemas intercomunales de vialidad regional, el planeamiento en extensión de determinadas zonas de destinación urbana y la confección de los planes reguladores y de abastecimiento regional de los sectores municipales de afinidad económica.

La posibilidad referida no había sido practicada, a pesar de la insistencia en ello de Muñoz Maluschka. Al asumir este planificador el cargo de jefe de la Sección de Urbanismo (c.1936), produjo el primer proyecto intercomunal indicativo para Santiago. En él dispuso un anillo de circunvalación actual Américo Vespucio, confirmó el Plan de Brunner para la comuna de Santiago y Providencia, y avanzó en la planificación de lo que hoy es Vitacura, y antes Las Condes ${ }^{10}$. Dejó, así, tácitamente establecida su discrepancia con Brunner tal vez la única respecto de la proyección para Santiago: Brunner supuso un millón de habitantes hacia 1960, lo cual se registró en los hechos en 1940. Hacia 1930 Chile tenía en $50 \%$ de la población urbana, por diversas razones históricas, aun cuando no estaba industrializado.

Es posible que la prioridad dada a Santiago, haya retardado el planteamiento intercomunal de Muñoz Maluschka para la intercomuna de Valparaíso ${ }^{11}$.

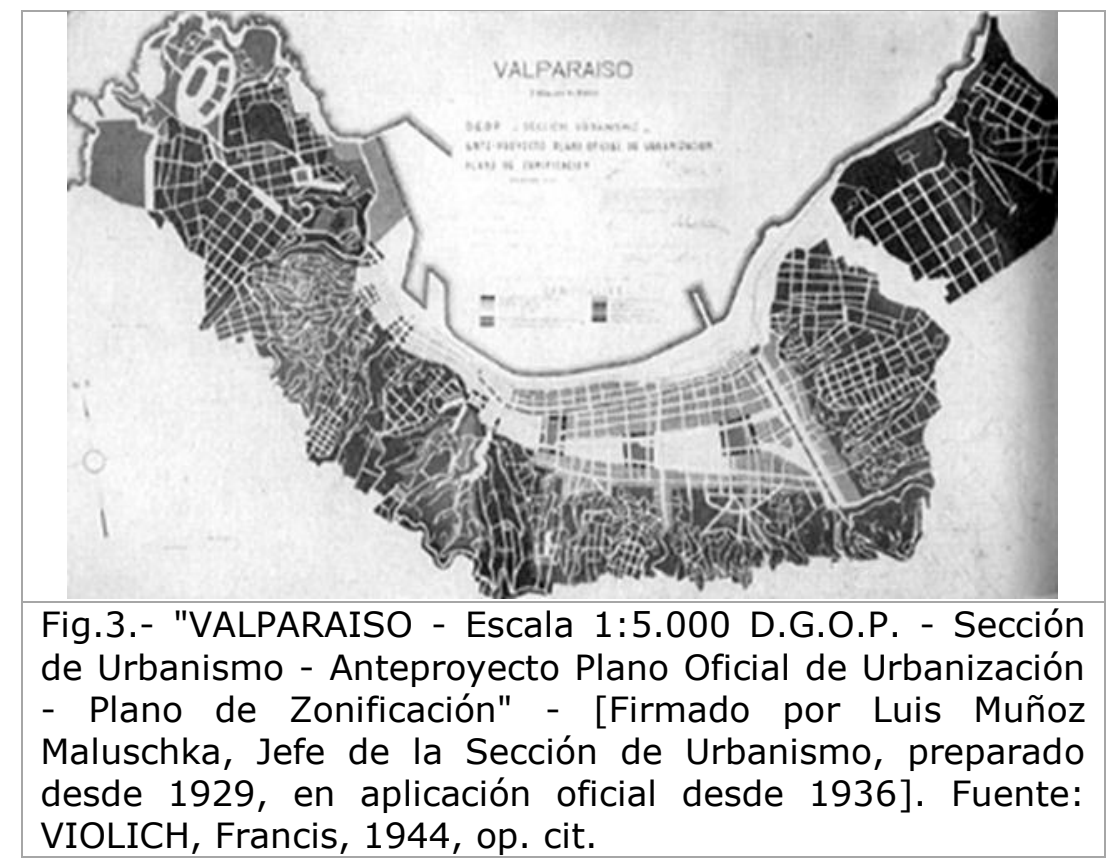

\footnotetext{
9 Hemos adoptado esta fecha, a partir diversas informaciones proporcionadas por el libro de Violich citado, pero es posible que la proposición pudiera ser de 1935.

10 Publicado en el libro de F. Violich, 1944, citado.

11 Desconocemos si hubo, posteriormente, un plano equivalente al de Santiago para Valparaíso-Viña del Mar.
} 
Aun cuando no hemos tenido a la vista el original de plano comunal "VALPARAISO D.G.O.P. - Sección de Urbanismo - Anteproyecto Plano Oficial de Urbanización - Plano de Zonificación ", queda en evidencia en la pequeña reproducción aportada por Violich, que el uso de suelo, la densidad de población y, sin duda, los restantes indicadores obligatorios al espacio privado, e incidentes en la configuración del espacio público y del paisaje urbano, ya no quedaban al azar en la época.

También es un hecho para nosotros, a la luz de nuestras investigaciones sobre tan famoso -aunque no leído arquitecto Luis Muñoz Maluschka ${ }^{12}$, que los proyectos o anteproyectos con su firma se basaron en un conjunto de conceptos que presentaron ciertas variantes respecto de los postulados en los Congresos Internacionales de Arquitectura Moderna, resumidos en la Carta de Atenas.

Una rápida confrontación de conceptos que hemos realizado a partir del conjunto de escritos de Muñoz Maluschka que hemos compilado, frente a las "exigencias" de la Carta de Atenas $^{13}$ - 39 puntos, de un total de 95 enunciados el pensador nativo presentan 12 discrepancias fundamentales; frente a los 25 puntos conteniendo "críticas y observaciones", se detecta 4 discrepancias más.

Conforme a las ponencias de nuestro especialista nativo, las zonificaciones deberían comprender usos mixtos compatibles toda vez que fuera posible, con lo que se desestimó el modo de uso especializado promovido de manera radical por el Movimiento Moderno; no se favoreció la separación de la circulación de peatones y vehículos drásticamente pues se tenía conciencia que especialmente en áreas centrales el esquema tradicional contribuía a la animación y al éxito comercial; no se desestimó el alineamiento de viviendas al borde de las calles ni la eventual existencia de formaciones urbanas tipo ciudad-jardín; más que la liberación de "grandes superficies verdes", se promovió el escalón de parque de juego de niños y adultos a escala del barrio (proximidad a la vivienda, con expresa referencia a su importancia para la regulación del microclima local); no se impidió tener micro-espacios verdes privados; el urbanismo fue visto como una ciencia de cuatro dimensiones y no sólo de tres, se estimó imprescindible el manejo del factor tiempo en Urbanismo, para realizar ahorros canalizando las demandas de uso también hacia el eje del tiempo; la ciudad se hace primero desde la Planificación Urbana y no desde la arquitectura. La movilización del suelo urbano se realizará sólo puntualmente en función del interés colectivo. Luis Muñoz, junto a otros urbanistas nativos de la época, reconocieron por otra parte, la diversidad de los grupos sociales en las ciudades y el país; la diversidad de las circunstancias de vida coexistiendo, la diversidad de latitudes en nuestro territorio habitable, por lo cual rechazaron la estandarización de los modos de vida y de la vivienda.

Luis Muñoz promovió, por otra parte, coeficientes de aprovechamiento de los terrenos limitados en relación con el valor económico de las zonas urbanas; la ocupación adecuada y oportuna de los espacios eriazos al interior de las ciudades antes de crecer

\footnotetext{
12 PAVEZ REYES, M. Isabel (compiladora). 1993. Luis Muñoz Maluschka, Escritos, D. Urbanismo, F.A.U., Universidad de Chile, noviembre de 1993, 86 p.

PAVEZ REYES, M. Isabel. 2003. Vertientes Urbanísticas Modernas en Chile 1929-1959: Conceptos de Vialidad y Transporte en los debates y estrategias de ordenación del territorio. T.T. P. Doctoral U. Politécnica de Madrid - U. Chile. P. Guía Dr. Ing. Julio Pozueta E., 204 p. ilus.

13 LE CORBUSIER, Principios del Urbanismo (La Carta de Atenas), Barcelona, Editorial Ariel, Esplugues de Llobregat, 1971/73.
} 
por extensión, por los costos que esto significa en redes, equipamientos y ocupación del suelo agrícola; mejor aprovechamiento del suelo urbano elevando moderadamente la altura de la edificación residencial y organizando zonas de pareos continuos; la implantación de la jornada única de trabajo con un término diferenciado de las labores en las distintas actividades en las grandes ciudades, para evitar congestiones innecesarias y reducir las expropiaciones para ensanches viales; realización de investigaciones científicas interdisciplinarias que dejaran en evidencia la relación entre la cultura humana y su expresión en los conjuntos arquitectónicos, entre otros ${ }^{14}$.

En tanto, en el nivel local de la administración, el arquitecto Ricardo González Cortés tuvo la iniciativa de invitar a la Municipalidad de Santiago, al urbanista francés Jacques Lambert. El anuncio de esta visita provocó mucho entusiasmo entre los arquitectos interesados en el Urbanismo en Chile. Entre otros aportes, Lambert colaboró durante su permanencia en Santiago, con la Comisión que preparaba con la participación de Karl Brunner la Ordenanza de Construcciones y Urbanismo, donde se intentó fortificar la acción de los municipios, dotándolos de algunos instrumentos necesarios a su acción.

\section{Jacques Lambert en Chile, en 1929}

La trayectoria y realizaciones de Jacques-Henri Lambert (n.1890-s/d) remiten, en primer lugar, fuera de Francia, pues cumplió misiones para el gobierno francés en los U.S.A. en 1922-29 (New York, Chicago, Washington), en Grecia, Egipto y Oriente Medio, en 1931; Turquía, en 1933 y 1940, Región de París, en 1932-35; en México en 1929-31-32; en Chile y Argentina en 1929-30; en Venezuela colaborando con el Ing. M. Rotival, en 1937-39. Trabajó también en misiones en Europa del Norte y Central ${ }^{15}$. En 1941 fue llamado a trabajar en el Plan de Lyon $^{16}$.

En la experiencia francesa, en la cual se inscribe Lambert, se llamó "urbanismo" al movimiento preocupado de embellecer, higienizar y organizar la extensión de las

\footnotetext{
14 Luis Muñoz, un "moderno reflexivo", realizó una relevante transferencia cultural en Urbanismo desde Alemania donde sus interlocutores fueron Werner Hegemann y Kurt Brüning. Luego de 20 años de intercambios con Alemania se le nombró "Miembro Extraordinario" por la Akademie für Raumforschung und Landesplanung, A.R.L.

15 FORMA URBIS, Les plans géneraux de Lyon XVIe-XXe siècles. [En línea] http://www.archivesIyon.com/fonds/plan-g/p27.htm

Forma Urbis recomienda ver sobre Lambert, en especial a D. BERTIN, "Grande opération au nord des Terreaux par l'ingénieur Jacques Henri Lambert (1941-1946)", en BULLETIN DE LA SOCIÉTÉ HISTORIQUE, ARCHÉOLOGIQUE ET LITTÉRAIRE DE LYON, t. XXII (année 1992), Lyon, 1993, pp. 41-46, 6 ill. en noir.

16 El primer "plan de embellisssement et d'extensión" de Lyon fue presentado en 1914, una obra de "aficionados iluminados" que presentó un esquema de mejoramiento de las vías públicas considerando proposiciones previas desde 1900. La Guerra y la Ley Cornudet modifican en parte estas primeras reglas del juego; en 1935 aún no se logra aprobar un Plan. El gobierno francés tutelado por los alemanes rediseñará el paisaje francés. En febrero de 1941 se crea la Delegación Gral. del Equipamiento Nacional, encargada de regionalizar el territorio y también de luchar contra la cesantía. Los ingenieros de Puentes y Caminos reconstruyen la infraestructura vial de Francia en sólo dos años. Sin embargo, un arquitecto es necesario para un plan de Lyon "digno". Jacques Lambert (57 años) asume la tarea desde el 28 de octubre de 1941. Su nombre estaba en las listas de profesionales recomendables (octubre-diciembre de 1940, "capaz de ocupar las funciones de urbanista jefe"). Formado en Beaux Arts, hacia 1940 aún no es miembro de la Soc. Francesa de Urbanistas. No es una figura mayor del Urbanismo en Francia, sino un experto en planificación urbana y diseño de parques y jardines más bien nómade. Al comenzar 1944, Lambert presenta al Concejo Municipal sus proyectos, pero este deseaba regresar a la situación de antes de la guerra. El contrato de Lambert fue anulado, su plan se descartó por ser demasiado "espectacular", y el servicio de arquitectura y urbanismo fue disuelto. Se retomará en 1947 el plan de Lyon, considerando también aquello del plan Lambert que pudiere ser factible, y los avances de 1935. (SAUNIER, Pierre-Yves, 1997, op. cit.).
} 
ciudades; de establecer las reglas para un ordenamiento y funcionamiento racional de ellas; de reconocer las leyes de la urbanización y del crecimiento; de instaurar una gestión científica de las ciudades, pero también, de trabajar sobre las comunidades y las sociedades de la era industrial ${ }^{17}$.

Verdaderos militantes intentaron instaurar una disciplina científica, un aparato legislativo, una acción pública y, aún, una profesión, aunque no tenían la misma concepción respecto de lo que trataban de establecer. Para ellos, tanto como para sus afines británicos, alemanes, holandeses, belgas, italianos o americanos que frecuentaban en los congresos y exposiciones que se multiplican en los primeros años del siglo $\mathrm{XX}$, el plano fue el soporte, el útil y el objetivo a la vez ${ }^{18}$. Más que por los diagramas, el dibujo, el gráfico, la tabla conteniendo la estadística, o la maquette, es mediante el plano que los reformadores de lo urbano quisieron comprender, actuar y comunicar sus aportes, fue alrededor de él que se hicieron los acuerdos y las oposiciones, mediante él se guió el desarrollo futuro de las ciudades ${ }^{19}$.

Gracias al plano, presentado en diversas escalas, era posible luchar contra los desafíos impuestos por la ciudad de la era industrial. El plano fue un útil de previsión y de control para comunas de más de 10.000 habitantes con la ley de 1919-1924, Ilamada "Ley Cornudet" (la "primera Carta del Urbanismo", según Gastón Bardet). Estas comunas debían contar en adelante con un plano "d'aménagement, d'embellissement et d'extension" realizado por un "homme d'art" ${ }^{20}$. Los planos eran objetos para especialistas y administradores que los utilizaron como propaganda; se les exponía, se les difundía, se les publicaba.

En los documentos que Lambert aportó a los urbanistas chilenos en $1930^{21}$ señaló que concebir con amplitud no bastaba, era necesario ejecutar también con amplitud dentro de las posibilidades financieras de cada ciudad. Era conveniente pues, mirar no sólo el presente, sino también un poco más adelante ${ }^{22}$. Rechazó las vías diagonales refiriéndose a aquellas verdaderas diagonales y no a vías, radiantes $u$ otras en aquellas ciudades cuyo plano formaba un tablero de ajedrez, o, por lo menos estimaba conveniente reducirlas al mínimo por cuanto esta clase de vías deja a ambos costados

17 SAUNIER, Pierre-Yves, 1997. "Au service du Plan: Hommes et structures de l'Urbanisme a Lyon au 20e siècle". En: FORMA URBIS. Les plans généraux de Lyon du XVIe au Xxe siècle, Lyon, Archives Municipales de Lyon, 1997, 19 p.

18 SAUNIER, Pierre-Yves, 1997, op. cit.

19 Destaca SAUNIER que la mística de los planos como instrumento de comprensión de los fenómenos sociales había sido bosquejada los "estadísticos sociales" de los años 1830, difundida en los años 1850 por las grandes exposiciones universales y los trabajos de Frédéric LE PLAY, y adoptada con entusiasmo por los reformadores urbanos británicos, entre ellos Patrick GEDDES, este último apreciando en la cartografía un vector privilegiado del progreso de la ciencia moderna. Aun cuando en Francia habrá que esperar hasta 1940 para que la cartografía social se vuelva un instrumento privilegiado de la investigación y de la acción administrativa y política, los urbanistas franceses habían escuchado los consejos de sus colegas británicos. Léon JAUSSELY (1875-1933) y sus colegas de la Sociedad Francesa de Arquitectos Urbanistas en Comment reconstruire nos cités détruites? (1915), hicieron del plano el medio principal de la transmisión de los resultados de las encuestas previas a la elaboración de los "planes de conjunto" para el ordenamiento y la extensión de las aglomeraciones urbanas y rurales. Ellos siguieron en esto las recomendaciones de R. UNWIN, quien en Town planning in practice (1909) aplica las consignas de Patrick GEDDES: todos los resultados de las encuestas deben ser traducidos en los planos. (SAUNIER, Pierre-Yves, 1997, op. cit.).

20 SAUNIER, Pierre-Yves, 1997, op. cit.

21 LAMBERT, Jacques H., (fecha desconocida entre marzo de 1929 y marzo de 1930). "Apuntes sobre Urbanismo". En: Arquitectura y Arte Decorativo, N09, Órgano Oficial del Colegio de Arquitectos de Chile, Santiago de Chile, marzo de 1930, pp. 397-399. [Traducido, o hecho traducir, por Ricardo González Cortés]. 22 Ibidem. 
de ellas parcelas de terreno de forma triangular de una utilización dificultosa, o terrenos en forma de estuche de muy difícil aprovechamiento. Sugería utilizar en lo posible las vías existentes, mejorándolas si era necesario, y uniéndolas en tal forma que llegaran a constituir circuitos, los que circundando los barrios y combinándolos con radiantes bien estudiadas, permitirían recoger, encauzar y dirigir el tránsito.

Lambert consideró primordial tratar cuidadosamente el tema de los accesos a las ciudades, primero, por lo que toca a la ciudad en sí misma en relación con sus suburbios y el aeropuerto, y también para permitir que los flujos fueran canalizados en forma constante hacia los puntos de importancia de la aglomeración. En toda ciudad moderna, su centro así como también sus principales vías y las de acceso, debían estar concebidos y estudiados para el automóvil, ya que éste estaba suplantando al peatón en el dominio de la calle. Se debía evitar la ejecución de plazas y encrucijadas de dibujo atrayente en el plano pero que, en la práctica, tendrían poca adaptabilidad a las necesidades del tránsito.

En cuanto a las directivas del urbanista éstas debían ser, según Lambert, la razón, el raciocinio y el desinterés; nunca prestarse para combinaciones o negocios inmobiliarios, se debía recoger y examinar todas las sugerencias que se le hiciera, reteniendo únicamente aquéllas que estuvieran conformes con los intereses de la colectividad. Por otra parte, el urbanista debía evitar la concepción de proyectos demasiado grandes pues se correría el riesgo de no poder realizarlos, produciendo al final un engaño con el solo resultado de un beneficio moral transitorio para el autor.

Se debía estudiar la situación de cada ciudad, su estado sanitario, el funcionamiento de todos y de cada uno de sus organismos, y todo esto con el espíritu crítico más imparcial; se trataría de aplicar todos los conocimientos técnicos, y el fruto de la experiencia.

Señalaba Lambert que el snobismo y la moda, no están ausentes del Urbanismo. Por ejemplo: la moda de las diagonales que dominaba aún la opinión pública latinoamericana, y de los grandes espacios abiertos indiferenciados cubiertos de césped, sin sombra, de una manifiesta inutilidad y de gran costo de mantenimiento. También detectaba Lambert en Latinoamérica el deseo de transformar lo que existía y estaba bien hecho, cuando muy cerca existían espacios libres o abandonados en los cuales se podrían desarrollar ideas nuevas, monumentos o jardines.

Debía evitarse el destrozar continuamente un determinado barrio, alterando su vida por caprichos de orden estético o pseudo estético, ya que los esfuerzos hechos por los comerciantes para mejorar sus locales y aumentar sus negocios, o por los propietarios para valorizar sus propiedades son dignos de consideración, interés y respeto, en la medida en que estos intereses concuerden con el interés general de la ciudad. Por tanto, debía reducirse al mínimo las operaciones quirúrgicas, tales como aperturas de diagonales, o de calles nuevas y de plazas que no tuvieran el carácter de urgencia absoluta. En todo caso, se debía evitar a todo trance desplazar o destruir los árboles, los monumentos de valor histórico o estético, o simplemente pintorescos dentro del paisaje urbano.

Coincidiendo con Muñoz Maluschka, consideró Lambert que todas las operaciones de urbanismo, ya fueran modificaciones, ensanches o extensión de calles, aperturas de 
nuevas vías, creación de cinturas, parques, etc., sólo serían provechosas cuando formaran parte de un plano intercomunal, con el entendimiento de todas las Municipalidades interesadas, para lo cual sólo un "sindicato de comunas" sería capaz de abarcar la totalidad de las operaciones útiles a la colectividad ${ }^{23}$ [23].

Advirtió, finalmente, Lambert ${ }^{24}$ :

...nunca faltan los aficionados que después de haber leído gruesos tomos que tratan de estas cuestiones, se sienten animados de un gran entusiasmo y sueñan qué digo exigen para su ciudad las transformaciones más extraordinarias $y$ muchas veces las menos adecuadas a sus necesidades. Cada uno de ellos clasifica su ciudad a su antojo, los unos entre las ciudades industriales y ruidosas, los otros entre los sitios más ideales, predilectos de las musas, soñando para ellas calles medioevales, plazuelitas y jardincitos con sombras propicias para los enamorados y los poetas. A todas esta gente el urbanista la tendrá siempre contra él, ya que para los primeros no será sino un retardatario y para los otros un innovador peligroso, aun cuando haga lo posible por convencerlos a todos que sus intenciones son las más puras y que sólo persigue el objetivo de dotar a la ciudad del plano más propicio para su desarrollo natural.

Lambert acompañó su aporte con una serie de bosquejos y croquis que fueron mantenidos en reserva por la Dirección de Obras de la Municipalidad de Santiago aun cuando no se trataba de un proyecto pues se temía a las especulaciones e influencias de los intereses creados ${ }^{25}$.

\section{El concurso del Alcalde Rosas para la transformación de Valparaíso}

La presencia de Lambert en Chile, coincidió con la apertura de un concurso de ideas para la transformación de Valparaíso. La revista "Arquitectura y Arte Decorativo", órgano oficial de la Asociación de Arquitectos de Chile $(1929)^{26}$, celebró en su página editorial "Valparaíso ciudad de gran porvenir" la apertura de un concurso para el estudio de la transformación de la ciudad puerto; se estimaba que Valparaíso era la

23 LAMBERT, Jacques, op. cit.

24 Ibidem.

25 El ingeniero-arquitecto Carlos Carvajal hizo ver, a propósito de esto, que principalmente en Alemania y los U.S.A., los mejoramientos de ciudades eran estudiados por todas las entidades científicas del ramo, y el público discutía los proyectos desde todos los puntos de vista, "haciendo sanas observaciones" que la prensa diaria y profesional acogía y apoyaba muchas veces. Estimaba Carvajal que los Consejos o Institutos de Urbanismo, corporaciones técnicas e independientes, "formados por los más capacitados", eran los llamados a resolver esas cuestiones, a adoptar resoluciones resultantes de las ideas expuestas a favor de la ciudad. Se evitaría, así, que pudiera ejercerse la influencia de caudillos y dirigentes "por más respetables que fueran". Lamentaba, por otra parte, que en Chile no hubiera aún en la administración pública un organismo técnico que satisficiera estas necesidades. De allí, la oportunidad que encontraba en la formación de un Comité Central de Urbanismo. Fte.: CARVAJAL M., Carlos. 1929. "La transformación de Santiago", (Capítulos 1 y 2 , de cuatro), (A don Alberto Mackenna Subercaseaux, Presidente del Comité Central de Urbanismo (julio de 1929). En: ARQUITECTURA Y ARTE DECORATIVO, No 6 y 7, Órgano Oficial de la Asociación de Arquitectos de Chile. Número especial Exposición Sevilla y Barcelona, Santiago de Chile, octubre de 1929, pp.271284/339-388. / CARVAJAL M., Carlos. 1929. "La transformación de Santiago", (Capítulos 3 y 4, de cuatro). (A don Alberto Mackenna Subercaseaux, Presidente del Comité Central de Urbanismo (julio de 1929). En: ARQUITECTURA Y ARTE DECORATIVO, No 8 y 9, Órgano Oficial de la Asociación de Arquitectos de Chile, Santiago de Chile, diciembre de 1929 - marzo de 1930, pp. 339-348/383-388.

26 ARQUITECTURA Y ARTE DECORATIVO, N³. "Valparaíso, ciudad de gran porvenir" [página editorial], Santiago de Chile, marzo de 1929. 
ciudad chilena donde podría aplicarse "con mayores posibilidades de éxito los más atrevidos sistemas modernos del Urbanismo".

"Su situación en plan y cerro, la disimetría de sus calles tortuosas en plano inclinado y gradas, llenas de rincones pintorescos, de sorpresas visuales, todo espera el talento de algún urbanista, verdaderamente conocedor de los problemas económicos, sociales y estéticos de una ciudad moderna, para descubrir y poner en relieve sus bellezas insospechadas.

Visto bajo ese aspecto del urbanismo, Valparaíso tiene una gran ventaja sobre Santiago, cuyo plano cuadriculado y sus calles interminables descartan toda sensación de improviso. Los terrenos disimétricos, con diferencias de nivel, permiten igualmente las soluciones atrevidas, llamativas y bellas, imposibles de realizar sobre un terreno uniforme".

Se estimaba que las concepciones inspiradas "en aquel famoso Jaussely, el Arquitecto Urbanista por excelencia " -el que falleció tres años después- podían dar los grandes rasgos del Valparaíso nuevo, con un horizonte de tiempo de cincuenta años [1980].

Para las proposiciones más detalladas, se confiaba en ser asesorados "por un buen técnico europeo como Jacques Lambert", a partir de las cuales los arquitectos e ingenieros chilenos podrían realizar ampliamente las modernas ideas que suponía la convocatoria al concurso para la transformación de Valparaíso.

El concurso solicitó seis estudios y proyectos, los que deberían dar lugar a obras que no podrían superar los 30 millones de pesos $(1929)^{27}$, a saber:

- $\quad$ Estudio de las vías de acceso a los cerros, planes de urbanización de los barrios pobres de la ciudad, con indicaciones de los perfiles tipo, pendientes más convenientes, vías de comunicación fácil entre los diversos cerros, etc.

- Transformación o mejora del plano de la ciudad, teniendo principalmente presente el problema de la vialidad para el fácil desarrollo comercial.

- $\quad$ Estudio de saneamiento, higienización, alumbrado, desagües, etc., de barrios o cerros aislados, en especial de los cerros Barón, Lecheros, Larraín, Recreo, Polanco, Molino, Toro, Arrayán, etc.

- $\quad$ Fijación de los límites urbanos de la ciudad.

- $\quad$ Proyectos de plazas, de un paseo a la orilla del mar, ubicación más conveniente y proyectos de plazas de deportes, juegos infantiles, etc.

- Indicaciones con mejoras estéticas de sitios especiales de la ciudad, tales como la Plaza Aníbal Pinto y otros, proyecto para la construcción de un nuevo Parque al lado del Camino de Cintura, en el cerro Bellavista ${ }^{28}$.

\footnotetext{
27 ARQUITECTURA Y ARTE DECORATIVO, N³. "A los ingenieros y arquitectos del país", Santiago de Chile, marzo de 1929, p. 86.

28 Miembros del jurado Ings. Enrique Middleton y Gregorio Airola, y el Arqto. Alberto Cruz Montt.
} 


\section{Labor del Instituto de Urbanismo de Valparaíso ${ }^{29}$}

La principal preocupación del Instituto fue en los años 1930, obtener que las municipalidades de las provincias de Valparaíso y Aconcagua cumplieran con la obligación legal de confeccionar sus planos reguladores. Es así que, en agosto de 1934 se celebró una de las más importantes reuniones de la época para intercambiar ideas al respecto. Los Arqtos. Agostino Bastiancig (Municipalidad de Viña del Mar) y el Jefe de la Sección de Urbanismo Arqto. Luis Muñoz Maluschka, hicieron una detallada exposición de la importancia de los planos reguladores y la forma de realizarlos. Otras iniciativas condujeron a la regularización de las Plazas Vergara, en Viña del Mar, y Brasil, en Limache. También de la plaza Sotomayor en Valparaíso, proporcionando además una entrada principal en su parte marítima digna de la importancia del puerto. Se consideró la construcción de los edificios Estación Puerto y Superintendencia de Aduanas, con dos torres simétricas que sirvieran de apoyo a una gran puerta de entrada.

En 1934 también se estudió el traslado de la vía férrea dividiendo a la ciudad de Viña del Mar con peligro para sus habitantes.

En julio de 1937 el Instituto convocó a una reunión con la concurrencia de autoridades centrales y edilicias, parlamentarios, y funcionarios públicos entre otros, con la finalidad de abordar un plan de obras para Valparaíso que solucionara radicalmente los problemas de vialidad y edificación de Valparaíso. La comisión estudió el anteproyecto presentado por el miembro del Instituto, Arqto. Agostino Bastiancig, el que se recomendó conjuntamente con un estudio de los abogados Alex varela y Héctor Vigil, enviando sus conclusiones al Alcalde de Valparaíso en 1938.

También el Instituto organizó el Primer Congreso Chileno de Urbanismo, el que se realizó entre los días 17 y 20 de febrero de 1938.

En marzo de 1939, la Municipalidad de Valparaíso, nombra una comisión para realizar su plan regulador oficial, y la Dirección General de Obras Públicas encarga el Arqto. Italo Sasso su confección bajo la dirección de la Sección de Urbanismo del Ministerio de Fomento.

En tanto, no estuvieron ausentes los aportes desde las Universidades al desarrollo de Valparaíso. Algunas imágenes del A. Proyecto de Título de David Cunco, (Prof. Guía Alfredo Johnson, U. s/d, c. 1938) con un proyecto de urbanización de la zona Estación Barón, han quedado registradas en revista Urbanismo y Arquitectura Nº6 (1940).

\footnotetext{
29 URBANISMO Y ARQUITECTURA N6, 1940, Santiago de Chile, Órgano Oficial de la Asociación de Arquitectos de Chile, pp. 33-40; p.66.
} 


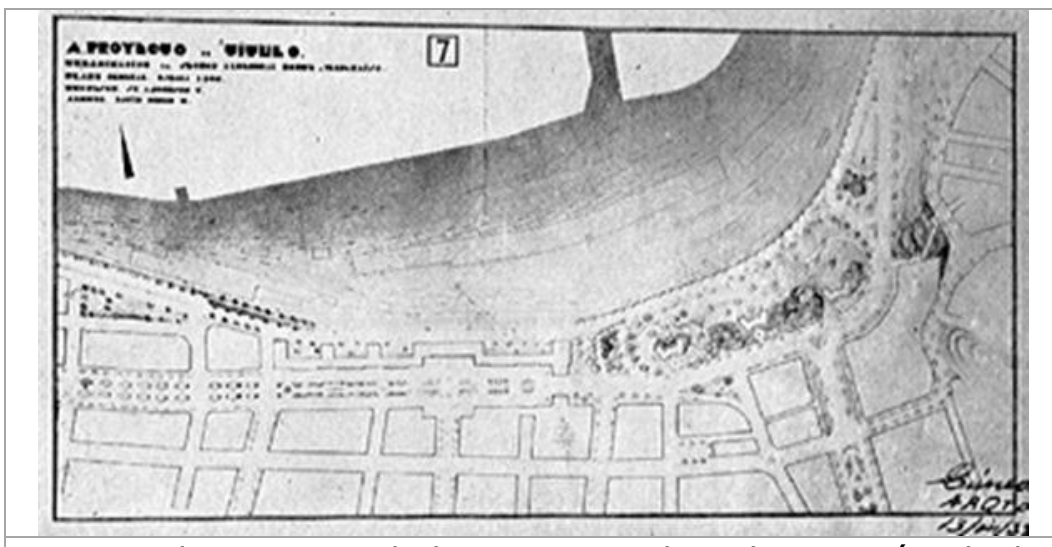

Fig.4.- Plano general de proyecto de urbanización de la zona Estación Barón, por David Cunco. Fuente: URBANISMO Y ARQUITECTURA N6, 1940, op. cit.

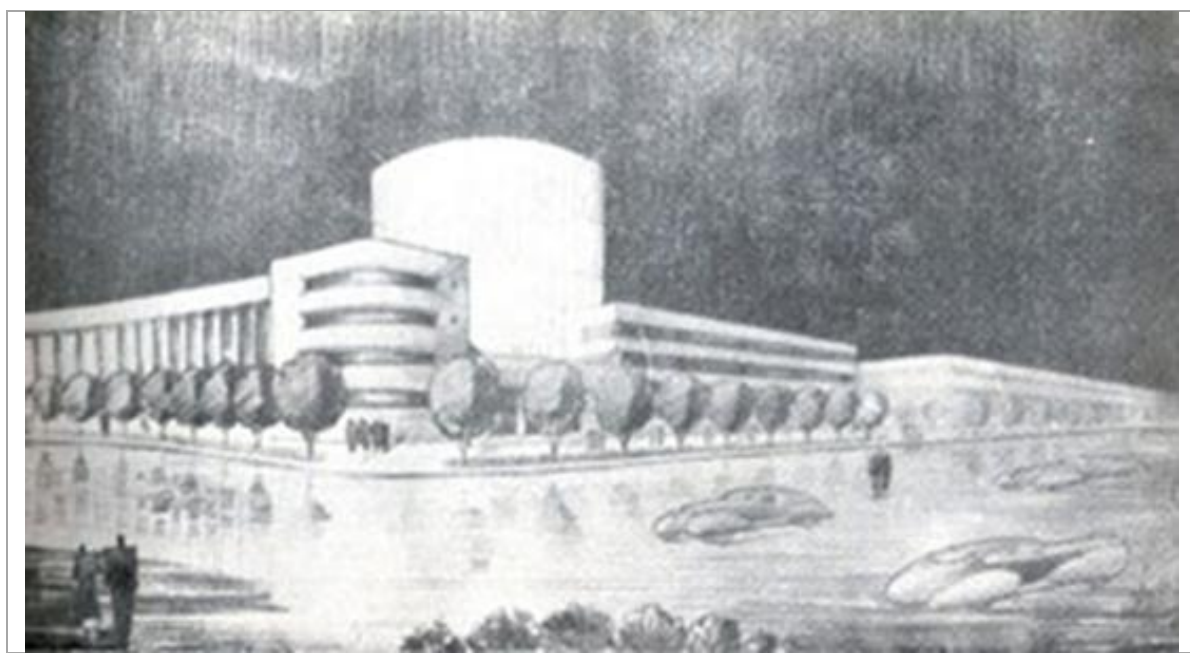

Fig.5.- Fachada del edificio principal en zona de urbanización Estación Barón, por David Cunco. Fuente: URBANISMO Y ARQUITECTURA Nº6, 1940, op. cit. 


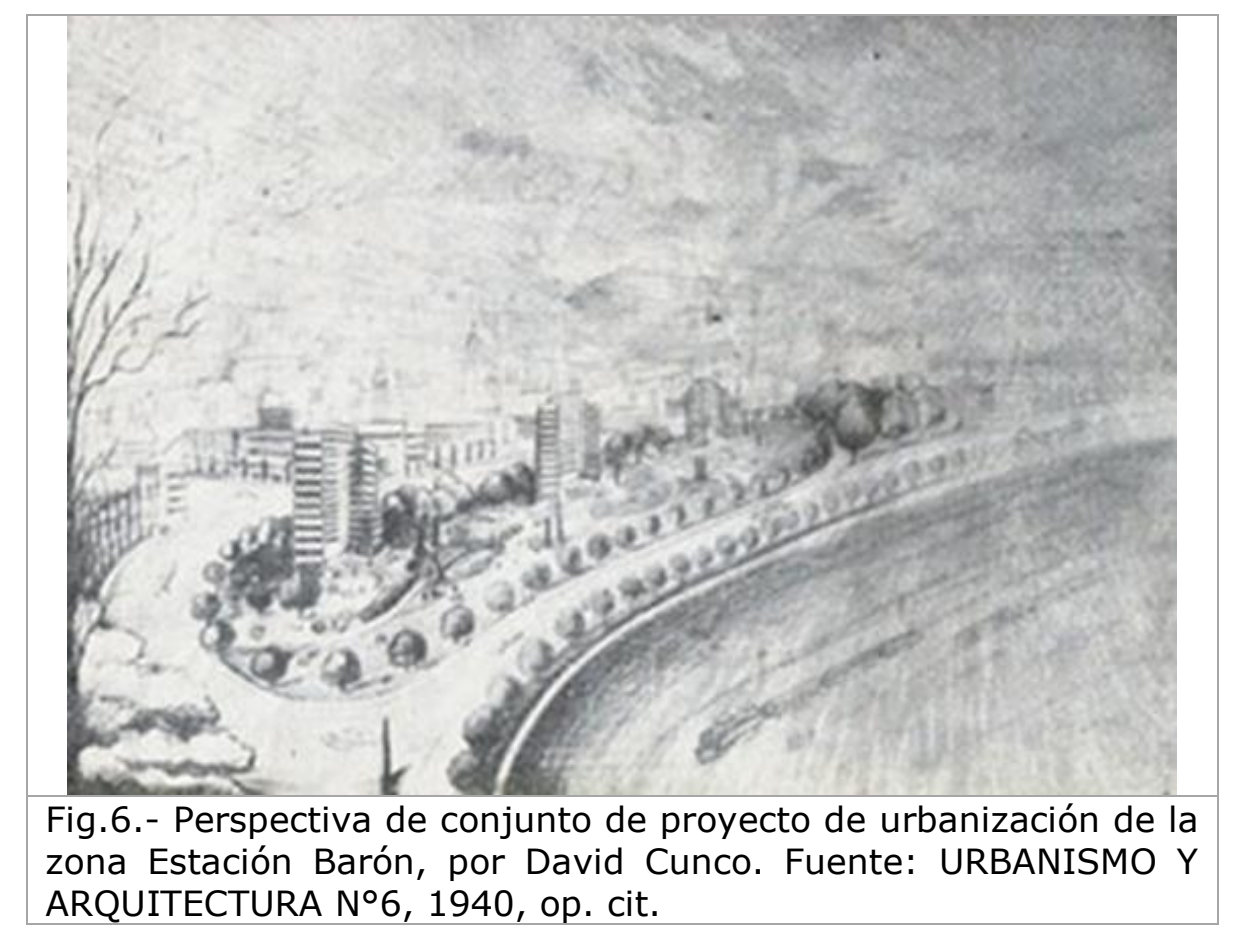

Por otra parte, los Arqtos. Browne y Valenzuela registraban su proyecto de urbanización en Caleta Abarca y Miramar.

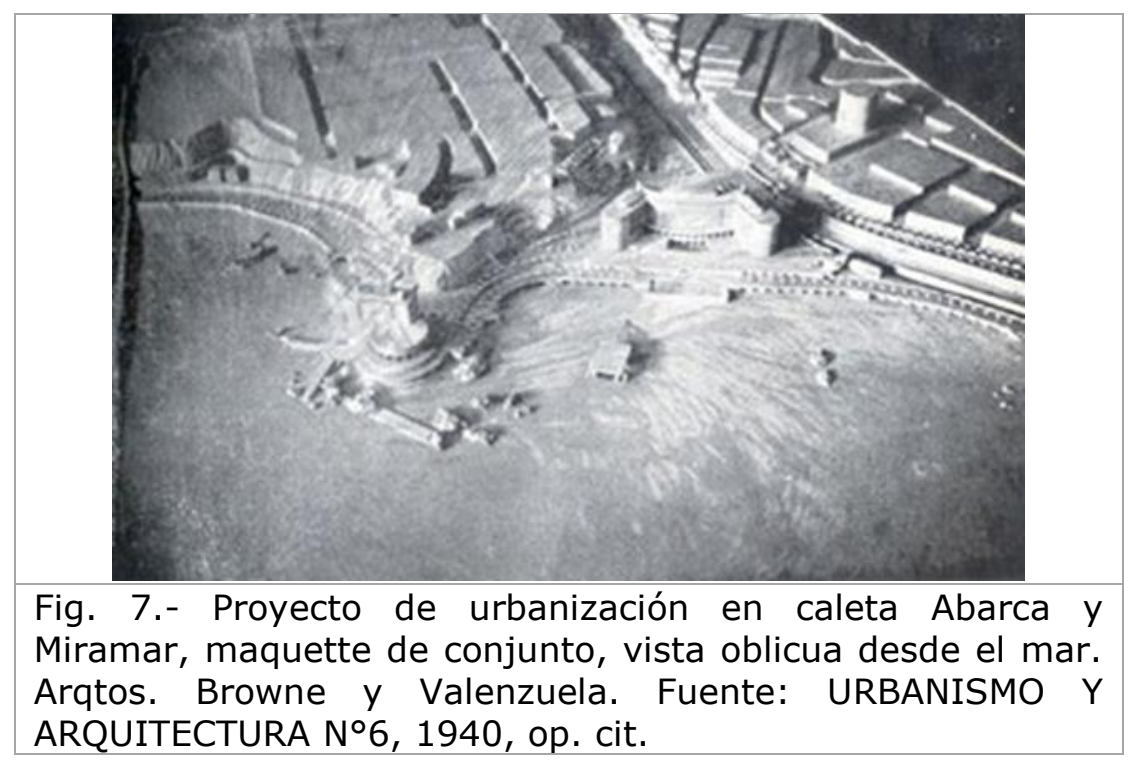




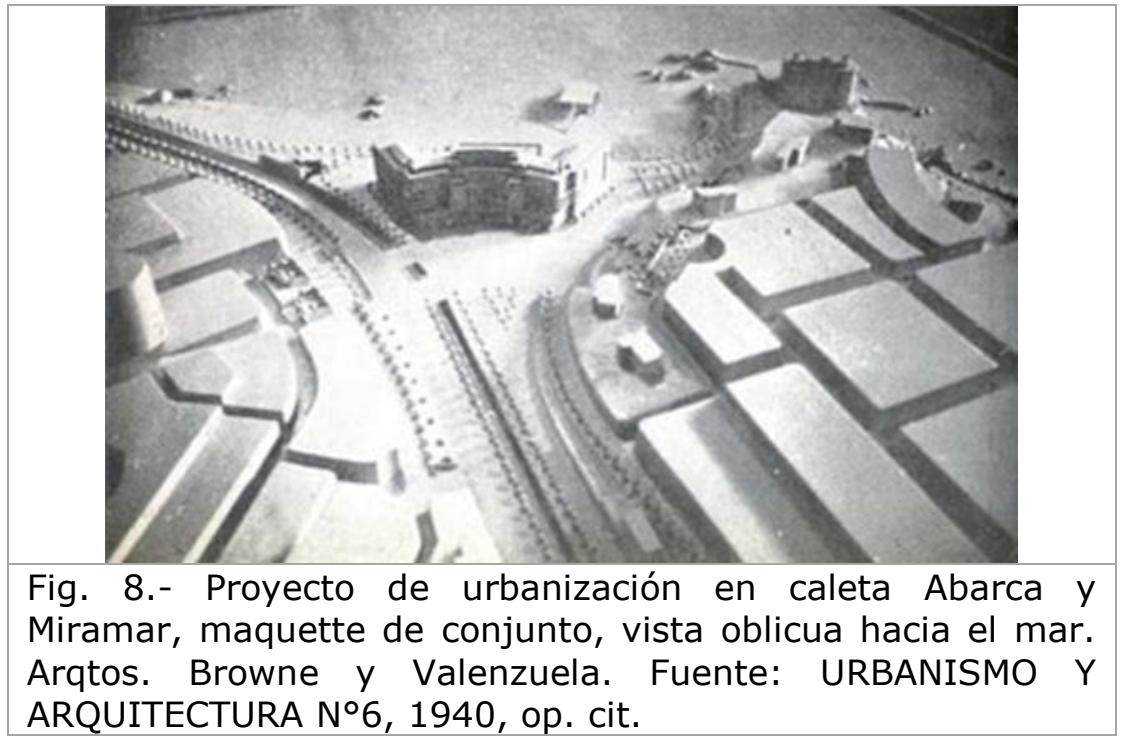

\section{Valparaíso se renueva o muere: proposición de "espirovías" en la Perla del Pacífico}

En 1937, y bajo el título sentencia: "Valparaíso se renueva o muere", el ingeniero, arquitecto y urbanista austriaco, Agostino Bastiancig Furlan ${ }^{30}$, residente en Viña del Mar, publicó, un sistema vial para facilitar la movilidad de los habitantes en su paso desde los cerros al plan y viceversa ${ }^{31}$. Tal vez su creatividad se desencadenó luego de las recomendaciones de Lambert sobre la necesidad de asumir el advenimiento de la era del automóvil, o tal vez participó en el concurso de 1929, inspirado en algunos proyectos futuristas europeos de principios del siglo XX.

En cualquier caso, cuando en la parte alta de Valparaíso reinaba "el desorden más completo", el Arqto. Bastiancig temía que las epidemias, el alto costo de la vida y la desvalorización continua de los terrenos conducirían a la desmoralización de los habitantes, y al abandono de la parte "más bella y sana de la ciudad". Observaba el arquitecto que, en la época, los enfermos contagiosos tenían que ser transportados "junto con sus ropas infectas", un kilómetro en camilla por senderos muleros, bajándolos después por un ascensor público. Se preguntaba Bastiancig entonces por las consecuencias en caso de una epidemia en la ciudad.

Descartados los medios mecánicos, el estudio de los caminos corrientes era basado en la época en las premisas de que la gradiente no debía ser superior a $5 \%$, las calzadas no menores de $12 \mathrm{~m}$, y las curvas de un radio no inferior a 40m, debiendo ocuparse la

\footnotetext{
30 Nació en Austria en 1883, estudió en la Real Esc. Industrial Superior de Trieste, y en el Real Politécnico y en la Academia de Bellas Artes de Viena. Miembro de The City Garden Association de Londres e Instituto de Urbanismo de Chile. Prof. Jefe de la Sección Construcciones, y de la Sección Ebanistería de la U. Santa María. Miembro de la Sección Geofísica del Observatorio El Salto, del Directorio del Inst. de Urbanismo y de la Asociación de Arquitectos, Ingenieros y Constructores. (Diccionario Biográfico de Chile, Ed. E. Periodística de Chile, 1942, $4^{a}$ ed., p. 104).

31 BASTIANCIG, Agostino. "Valparaíso se renueva o muere". en: REVISTA ZIG-ZAG, Número Espacial Arquitectura, Construcción y Urbanismo, Stgo., dic. 1937, pp.98-99.
} 
menor superficie posible y desembocar lo más cerca del borde superior de los cortes de terreno que encierran la parte plana de la ciudad.

"Las calamidades que persiguen al primer puerto de la República tienen su origen en que el problema urbanístico no empieza con la zonificación como en los casos corrientes, sino que a la zonificación tiene que preceder la planificación, o sea, la transformación de las abruptas vertientes de los cerros y quebradas en terrenos cuya pendiente permita utilizarlos en beneficio de una función de la ciudad. A la planificación tiene que seguir el trazado de una red de comunicaciones que permita un cómodo y seguro acceso a los cerros y quebradas planificados. Estas soluciones implican un claro concepto de un problema tridimensional, cuya solución está lejos de los conceptos urbanísticos aplicados a las ciudades planas"32.

Según el planteamiento del Arqto. Bastiancig, esas condiciones se podían satisfacer proyectando un camino que, en forma de helicoide cilíndrica, se desarrolla sobre sí mismo, alcanzando el desnivel de 40 a $50 \mathrm{~m}$ necesario para superar los cortes del pie de los cerros. Resultaba de ello una torre de unos $100 \mathrm{~m}$ de ancho y unos $50 \mathrm{~m}$ de altura, en la cual se desarrollan dos calzadas independientes, sobrepuestas, una de subida y otra de bajada. El ancho de la calzada sería de unos $9 \mathrm{~m}$, el de las aceras de unos $3 \mathrm{~m}$, la altura entre calzadas de $6,50 \mathrm{~m}$ y el $5 \%$ de gradiente. Esas dimensiones permitirían el uso de carros eléctricos de adhesión.

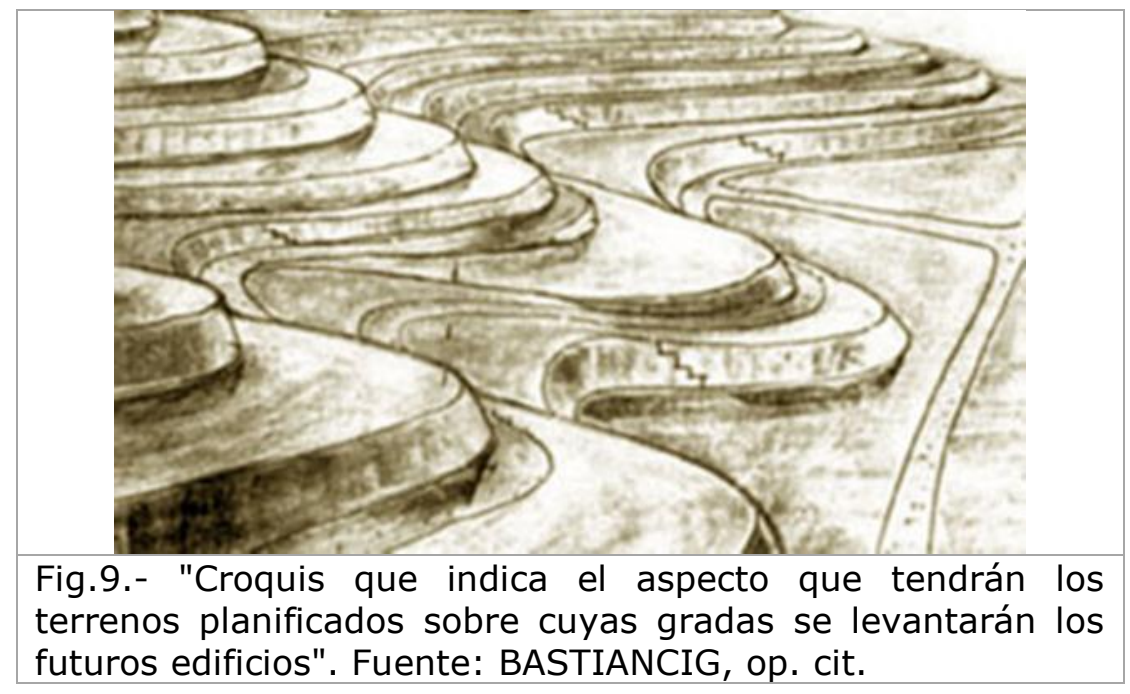

32 Ibidem. 


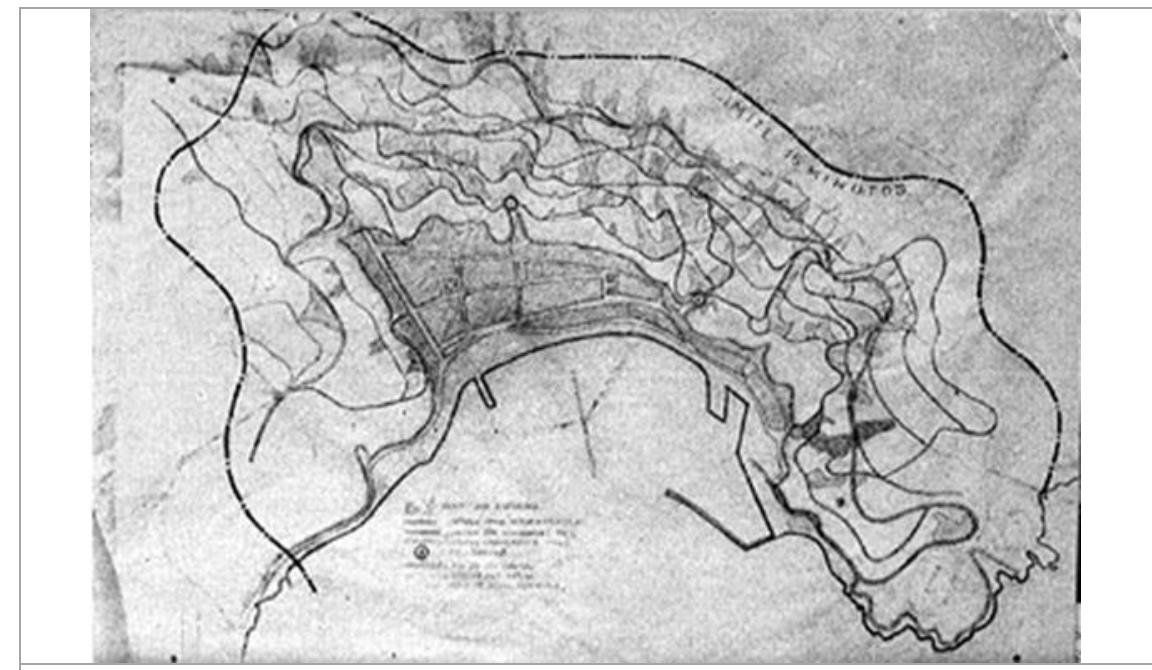

Fig. 10.- "Plano esquemático general, en el cual se indica la ubicación de tres 'Espirovías', de dos caminos de acceso directo sin 'Espirovía', el trazado de los caminos en los cerros; el trazado de comunicación de la parte alta con la retrotierra y Viña del Mar. Las superficies oscuras representan la parte plana ganada rellenando las quebradas. La línea exterior, a trazo y punto, indica el límite que se alcanzaría en 15 minutos desde el plan". Fuente: BASTIANCIG, op. cit.

La ubicación más conveniente para estas articulaciones que Bastiancig llamó "espirovías", sería a unos 50 u 80 m de la desembocadura de las quebradas en el plan, permitiendo, así, el acceso a dos cerros contiguos. Por este medio de comunicación, junto con caminos del $5 \%$ de gradiente, con cualquier vehículo corriente se podría alcanzar en 8 minutos, desde cualquier punto del plano, la cota 250-300 m sobre el nivel del mar. Recorriendo unos 7 minutos a pie, se alcanzaría el límite de la zona de 15 minutos que abarcaría una superficie de aproximadamente 1.500 hectáreas.

Conforme a soluciones italianas y alemanas, el Arqto. Bastiancig proponía que los caminos pasaran sobre muros que, además de servir de puentes, servirían para retener el material de relleno de las quebradas, cuya superficie arrojaría una 320 hectáreas planificadas. El relleno de las quebradas se haría con el material resultante de las excavaciones de gradas en los cerros, planificándolos según el recorrido de las curvas de nivel. Los desmontes resultantes se echarían en las quebradas y serían arrastrados por las lluvias al fondo de estas, depositándose en capas horizontales tras el muro de retención especial, que sólo dejaría filtrar el agua. La altura de los muros se aumentaría anualmente en relación con la altura del relleno. 

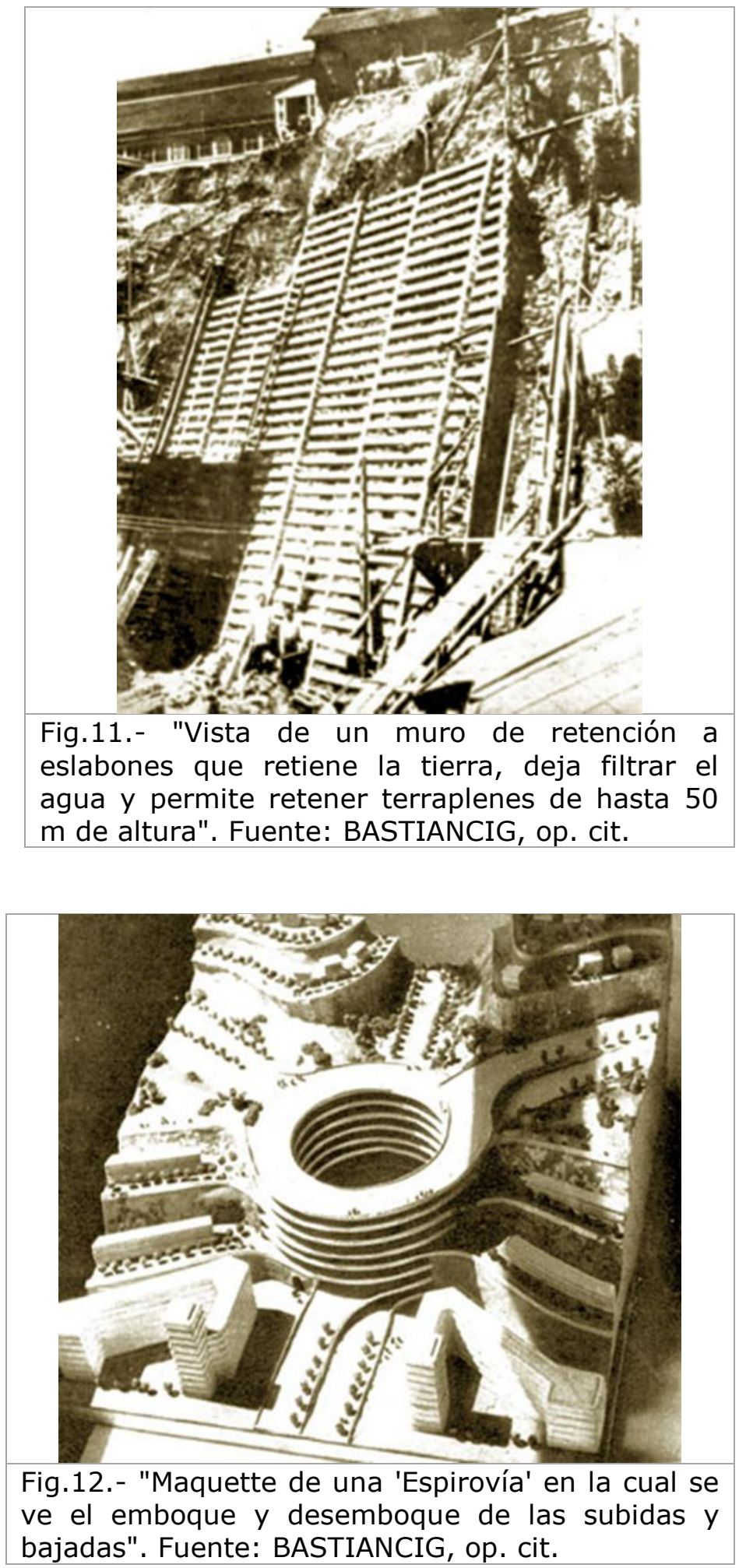


\section{Palabras finales}

Constatamos que, en el decenio de 1930, hubo importantes proyectos y ponencias referidos a la transformación de las principales ciudades chilenas, y una preocupación por solucionar algunos problemas particulares de Valparaíso. En esta acción apreciamos una capacidad reflexiva a partir de aportes internacionales, respecto de los cual los profesionales nativos fueron críticos toda vez que dichos aportes no presentaron coherencia con la realidad geográfica y económica del país.

Por otra parte, no faltó la mirada al sistema de centros poblados para efectos de una regionalización más adecuada, ni la mirada intercomunal toda vez que fue necesario, ni el ejercicio de creatividad en diseño urbano asociado a los objetivos de mejorar la conexidad, conectividad y nodalidad del sistema vial de Valparaíso, y con ello la calidad de vida de sus habitantes. Se indagó para ello en nuevos elementos originados en las condicionantes del sitio e incidentes en la nueva funcionalidad y legibilidad que iba requiriendo Valparaíso, "la Perla del Pacífico" en los momentos en que la industrialización del país se volvió una meta imperativa, y el advenimiento del automóvil un asunto insoslayable.

\section{Bibliografía}

ARQUITECTURA Y ARTE DECORATIVO, N³. "Valparaíso, ciudad de gran porvenir" [página editorial], Santiago de Chile, marzo de 1929.

ARQUITECTURA Y ARTE DECORATIVO, N³. "A los ingenieros y arquitectos del país", Santiago de Chile, marzo de 1929, p. 86.

BASTIANCIG, Agostino. "Valparaíso se renueva o muere". En: REVISTA ZIG-ZAG, Número Espacial Arquitectura, Construcción y Urbanismo, Stgo., dic. 1937, pp.98-99.

CARVAJAL M., Carlos. 1929. "La transformación de Santiago", (Capítulos 1 y 2, de cuatro), (A don Alberto Mackenna Subercaseaux, Presidente del Comité Central de Urbanismo (julio de 1929). En: ARQUITECTURA Y ARTE DECORATIVO, No 6 y 7, Órgano Oficial de la Asociación de Arquitectos de Chile. Número especial Exposición Sevilla y Barcelona, Santiago de Chile, octubre de 1929, pp.271-284/339-388.

CARVAJAL M., Carlos. 1929. "La transformación de Santiago", (Capítulos 3 y 4, de cuatro). (A don Alberto Mackenna Subercaseaux, Presidente del Comité Central de Urbanismo (julio de 1929). En: ARQUITECTURA Y ARTE DECORATIVO, $N^{\circ} 8$ y 9, Órgano Oficial de la Asociación de Arquitectos de Chile, Santiago de Chile, diciembre de 1929 - marzo de 1930, pp. 339-348/383-388.

CHILE - DIRECCION GENERAL DE OBRAS PÚBLICAS. 1930. Memoria anual correspondiente al año 1929, República de Chile, Talleres de la Editorial Nascimiento, 1930, 176 págs.

DiCCIONARIO BIOGRÁFICO DE CHILE, Ed. E. Periodística de Chile, 1942, $4^{a}$ ed. 
EL MERCURIO, Valparaíso, 27 nov. 1929. "El problema de urbanizar a Valparaíso tiene características propias que impiden seguir los métodos de otras ciudades".

FORMA URBIS, "Les plans géneraux de Lyon XVIe-XXe siècles". [En línea] http://www.archives-lyon.com/fonds/plan-g/p27.htm

INSTITUTO NACIONAL DE INVESTIGACIONES TECNOLOGICAS Y NORMALIZACIÓN, C.D.U. 69.05 + 711, 10 de noviembre de 1949, Ley y Ordenanza General sobre Construcciones y Urbanización. [Contiene compilación histórica de legislación precedente].

LAMBERT, Jacques H., (fecha desconocida entre marzo de 1929 y marzo de 1930). "Apuntes sobre Urbanismo". En: Arquitectura y Arte Decorativo, N09, Órgano Oficial del Colegio de Arquitectos de Chile, Santiago de Chile, marzo de 1930, pp. 397-399. [Traducido, o hecho traducir, por Ricardo González Cortés].

LE CORBUSIER, Principios del Urbanismo (La Carta de Atenas), Barcelona, Editorial Ariel, Esplugues de Llobregat, 1971/73.

MUÑOZ MALUSCHKA, Luis. 1936. "Planos Reguladores y zonificación territorial". En: revista ARQUITECTURA, Santiago de Chile, Editorial Antares, enero de 1936, $\mathrm{N}^{\circ} 4$.

PAVEZ REYES, M. Isabel (compiladora). 1993. Luis Muñoz Maluschka. Escritos, D. Urbanismo, F.A.U., Universidad de Chile, noviembre de 1993, 86 p.

PAVEZ REYES, M. Isabel. 2003. Vertientes Urbanísticas Modernas en Chile 1929-1959: Conceptos de Vialidad y Transporte en los debates y estrategias de ordenación del territorio. T.T. P. Doctoral U. Politécnica de Madrid - U. Chile. P. Guía Dr. Ing. Julio Pozueta E., 204 p. ilus.

SAUNIER, Pierre-Yves, 1997. "Au service du Plan: Hommes et structures de I'Urbanisme a Lyon au 20e siècle". En: FORMA URBIS. Les plans généraux de Lyon du XVIe au Xxe siècle, Lyon, Archives Municipales de Lyon, 1997, 19 p.

URBANISMO Y ARQUITECTURA N6, 1940, Santiago de Chile, Órgano Oficial de la Asociación de Arquitectos de Chile, pp. 33-40; p.66.

VIOLICH, Francis. Cities of Latin America. Housing and planning to the south. Nueva York: Reinhold Publishing Corporation, 1944. 241 p. 